\title{
Theme: Haematology
}

\section{Q}

A phase 3 randomized trial of voxelotor in sickle cell disease (NEngl JMed. 2019;381:509-19)

The two medications in regular use for sickle cell disease are hydroxyurea and L-glutamine. Inhibiting $\mathrm{HbS}$ polymerization in red cells can have a disease-modifying effect. The authors of this study aimed to evaluate the efficacy and safety of voxelotor, as compared with placebo, in adolescents and adults with sickle cell disease. A total of 274 patients were enrolled and randomly assigned in a 1:1:1 ratio to receive a once-daily oral dose of 1500 $\mathrm{mg}$ of voxelotor, $900 \mathrm{mg}$ of voxelotor, or placebo. The primary endpoint was the percentage of participants who had a hemoglobin response (an increase from baseline of $>1.0 \mathrm{~g} / \mathrm{dL}$ at week 24 ). In the $1500 \mathrm{mg}$ group, $51 \%$ achieved this endpoint compared to $33 \%$ and $7 \%$ in $900 \mathrm{mg}$ group and placebo, respectively. The increase in hemoglobin was concurrent with a reduction in markers of hemolysis, including indirect bilirubin, reticulocyte count and lactate dehydrogenase level. A trend of reduced incidence of vasoocclusive crises over time with voxelotor, as compared with placebo, was also observed. There was no difference in the frequency of adverse events in the three groups.

This study shows the way for a new disease-modifying agent that can reduce the morbidity and mortality in patients of sickle cell disease.

Prevalence of anemia among 6- to 59-month-old children in India: the latest picture through the NFHS4 (J Biosoc Sci. 2019 May 20:1-11. doi: 10.1017/ S0021932019000294. [Epub ahead of print])

India is one of the leading contributors to childhood anemia in developing countries. The authors aimed to assess the current anemia status in India using data from the most recent National Family Health Survey carried out in 2015-16. Data of 1,37,347 children aged 6-59 months living in 29 states and seven Union territories were analyzed. Overall, $56.3 \%$ of the children were anemic in $2015-16 ; 1.5 \%$ were severely anemic, $27.6 \%$ were moderately anemic, and $27.2 \%$ were mildly anemic. Areduction by $13.5 \%$ from NFHS-3 was noted. The percentage of anemia was $65.9 \%$ at $6-8$ months, increased to $68.1 \%$ at $12-23$ months, and then decreased to $42.7 \%$ at $48-59$ months. The North-east region was the least affected (34.6\%), and the Central region the most affected $(62.3 \%)$. The prevalence of anemia decreased in Northeast by $20 \%$ in 8 years, which was remarkable. Rural children were more anemic than urban children. Mother's education, mother's nutritional status, wealth status, and health status influenced the prevalence of anemia. It was also observed that the supply of IFA tablets was lower than expected, and many of the intended beneficiaries were unaware of the program.

This analysis reiterates the knowledge that besides iron supplementation, the overall education, nutrition and economic status have to be reformed to reduce the burden of anemia in Indian children.

INDIAN PEDIATRICS
WHO Global Initiative for Childhood Cancer ( $h t t p s: / /$ www.who.int/cancer/childhood-cancer/en/.)

Each year, more than 3,00,000 children aged birth to 19 years are diagnosed with cancer around the world. Approximately 8 in 10 of these children live in low- and middle-income countries where their survival rate is often near 20\%. In September 2018, WHO announced a new effort - the WHO GICC - to reach at least a $60 \%$ survival rate for children with cancer by 2030 , thereby saving an additional one million lives. This new target represents a doubling of the global cure rate for children with cancer. The aims of the initiative are two-fold: to increase the prioritization of childhood cancer through awareness raising at global and national levels, and to expand the capacity of countries to deliver best practice in childhood cancer care. Concretely, WHO will support governments to assess current capacities in cancer diagnosis and treatment, including the availability of medicines and technologies; set and cost priority cancer diagnosis and treatment programs; and integrate childhood cancer into national strategies, health benefits packages and social insurance schemes. SIOP (International Society of Pediatric Oncology) and the St. Jude Children's Research Hospital in the United States, have committed US\$ $15,000,000$ to support the implementation of the initiative.

Emicizumab versus factor VIII for prophylaxis in hemophilia A (Curr Med Res Opin. 2019;13:1-9)

Emicizumab is a humanized monoclonal bi-specific antibody that mimics activated Factor VIII, promoting the activation of downstream hemostasis at the site of bleeding in patients with hemophilia A without inhibitors. A half-life of 30 days and subcutaneous administration makes it an attractive prospect for prophylaxis. Researchers compared bleed rates in patients receiving emicizumab prophylaxis and patients receiving Factor VIII prophylaxis. They used data from a non-interventional study done before emicizumab and data from HAVEN 3 trial. A systematic review and network meta-analysis were also done to compare the same. Four studies that met the criteria were included. Prophylactic emicizumab was more effective than Factor VIII prophylaxis (RR 0.36; 95\% CI 0.13, 0.95). The new HAVEN 3 analyses also showed lower rates of treated bleeds with emicizumab prophylaxis than with Factor VIII prophylaxis.

US Food \& Drug Administration approved emicizumab in 2017 for the treatment of patients with hemophilia A with inhibitors and extended to patients without inhibitors in 2018. This study creates more evidence for the inclusion of emicizumab in the care of hemophilia A patients. However, long-term safety and accessibility to the drug will have to be considered before its inclusion in routine clinical practice.

EMINe A RAHIMAN AND * DEePAK BANSAL *deepakbansaldr@gmail.com

VOLUME 56-NOVEMBER 15, 2019 